\title{
Approximate design of cyclic electromagnetic drive with respect to permissible heating condition
}

\author{
V.Yu. Neyman \\ Novosibirsk State Technical University \\ Novosibirsk, Russian Federation \\ E-mail:nv.nstu@ngs.ru
}

\author{
L.A Neyman \\ Novosibirsk State Technical University \\ Novosibirsk, Russian Federation \\ E-mail: neyman31@gmal.com
}

\begin{abstract}
Impulse technologies application in cyclic electromagnetic machines demands improvement of thermal design procedures for the short-time mode. When such machines are developed, the thermal design procedures allow optimizing their operation with respect to the given working procedure. New design relations describing electromagnetic drive heating in the short-time mode have been derived from the finite-difference Newton equation solution. It is assumed that the electric drive is a homogeneous body with ideal heat conductivity. The approximate expressions of permissible heating with respect to impact energy depending on the number of operating cycles or impacts and initial temperature exceed over ambient temperature have been obtained. The derived dependences for the cyclic electromagnetic machine short-time mode can be widely used in practice. They will help to optimize electric drive operation with respect to the permissible heating condition when there is no need to use complicated mathematical formulas. A two-coil cyclic electromagnetic machine is considered as an example of using the design procedure for output parameters in the short-time mode.
\end{abstract}

Keywords - cyclic electromagnetic drive, initial temperature exceed, cyclic heating process, short-time mode

\section{NTRODUCTION}

Impulse technologies in industry are implemented by machines and units based on impact electromagnetic machines $[1,2]$.

Currently the impact electromagnetic machines are extremely improved and some of them are introduced into serial production [3].

Electromagnetic design methods are well-known and their improvement is going on [4-8].

New impulse technologies need improved engineering design procedures permitting to optimize an electric drive operation with respect to a given working procedure [9-12].

The electric drive cyclic mode with input power significantly more than power in the continuous running duty is considered in the paper.

When electric drive temperature achieves certain maximum permissible level, it is necessary to switch off and cool the drive.

When the electric drive is switched on again, initial temperature of it can exceed environment temperature.
Such control method with technological process interrupting permits to increase impact energy and electric drive power. This method is main one for a number of units.

The heating and cooling process in the electric drive cyclic mode for any $n$th cycle $(n=0,1,2 \ldots)$ is described by the Newton equations:

$$
\begin{cases}C \frac{d \tau}{d t}+k_{\mathrm{h}} S_{\mathrm{cs}} \tau=P_{\mathrm{L}}, & n t_{\mathrm{c}} \leq t \leq n t_{\mathrm{c}}+t_{\mathrm{cf}} ; \\ C \frac{d \tau}{d t}+k_{\mathrm{h}} S_{\mathrm{cs}} \tau=0, & n t_{\mathrm{c}}+t_{\mathrm{cf}} \leq t \leq(n+1) t_{\mathrm{c}},\end{cases}
$$

where $\tau$ is the electric drive temperature excess over the ambient temperature; $C$ is the electric drive heat capacity; $k_{\mathrm{h}}$ is the heat-transfer factor; $S_{\mathrm{cs}}$ is the cooling surface area; $P_{\mathrm{L}}$ is the heat loss; $t_{\mathrm{c}}=t_{\mathrm{cf}}+t_{\mathrm{cp}}$ is duty cycle time; $t_{\mathrm{cf}}$ is the coil current flow time; $t_{\mathrm{cp}}$ is the current pause time; $n$ is the number of cycles.

The electric drive load plot is stated in Fig. 1.

\section{CYCLIC ELECTRIC DRIVE APPROXIMATE DESIGN}

The equations system (1) solution in finite differences has the form:

$$
\begin{gathered}
\tau(n)_{\min }=\tau(0) \gamma^{n}+\frac{\tau_{\mathrm{s}}(1-a)\left(1-\gamma^{n}\right) \frac{\gamma}{a}}{1-\gamma}, \\
\tau(n)_{\max }=\tau(0) \gamma^{n}+\frac{\tau_{\mathrm{y}}(1-a)\left(1-\gamma^{n}\right)}{1-\gamma},
\end{gathered}
$$

where $\gamma=\mathrm{e}^{-\frac{t_{\mathrm{c}}}{T_{0}}}, a=\mathrm{e}^{-\frac{t_{\mathrm{cf}}}{T_{0}}}, T_{0}$ is the electric drive heating time constant; $\tau_{\mathrm{s}}$ is the overheating stationary value for longstanding power output; $\tau(0)$ is the initial temperature exceeding over the ambient temperature; $\tau(n)_{\min }, \tau(n)_{\max }$ are correspondingly the minimal and maximal overheating values in в $n$th heating working cycle. 


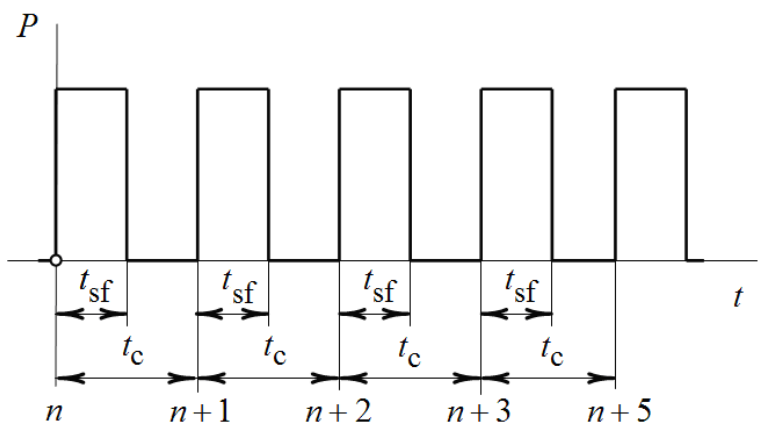

Fig. 1. Electric drive load plot.

The values $\tau(n)_{\max }$ and $\tau(n)_{\min }$ have not to exceed the given allowable value $\tau_{\mathrm{a}}$ :

$$
\tau(n)_{\max } \leq \tau_{\mathrm{a}}, \quad \tau(n)_{\min } \leq \tau_{\mathrm{a}}
$$

With respect to non-zero initial condition $\tau(0) \neq 0$ the expressions (2) and (3) are simply reduced to the form:

$$
\begin{aligned}
& \frac{\tau_{\mathrm{s}}}{\tau_{\mathrm{a}}}=\left[1-\frac{\tau(0)}{\tau_{\mathrm{a}}} \gamma^{n}\right] \frac{1-\gamma}{(1-a)\left(1-\gamma^{n}\right)} \cdot \frac{a}{\gamma}, \\
& \frac{\tau_{\mathrm{s}}}{\tau_{\mathrm{a}}}=\left[1-\frac{\tau(0)}{\tau_{\mathrm{a}}} \gamma^{n}\right] \frac{1-\gamma}{(1-a)\left(1-\gamma^{n}\right)} .
\end{aligned}
$$

The substitutions of the heat power overloading factor $\frac{\tau_{\mathrm{s}}}{\tau_{\mathrm{a}}}$ by $k_{\text {po }}$ and the relative initial temperature excess over the ambient temperature $\frac{\tau(0)}{\tau_{\mathrm{a}}}$ by $\varepsilon$ give:

$$
\begin{aligned}
& k_{\mathrm{po}}=\frac{\left(1-\varepsilon \gamma^{n}\right)(1-\gamma)}{(1-a)\left(1-\gamma^{n}\right)} \cdot \frac{a}{\gamma} \\
& k_{\mathrm{po}}=\frac{\left(1-\varepsilon \gamma^{n}\right)(1-\gamma)}{(1-a)\left(1-\gamma^{n}\right)}
\end{aligned}
$$

The expression (6) is suitable for minimal overheating value in the working cycle. The expression (7) is suitable for the maximal overheating.

The power overloading factor is:

$$
k_{\mathrm{po}}=\frac{P_{\mathrm{L}}}{P_{\mathrm{a}}},
$$

where $P_{\mathrm{a}}$ is the loss with respect to allowable heating from the condition of the long-time mode; $P_{\mathrm{L}}$ is the power loss in the time interval $t_{\mathrm{cf}}$.

The power loss is:

$$
P_{\mathrm{L}}=\frac{1}{t_{\mathrm{cf}}} \int_{0}^{t_{\mathrm{cf}}} i_{\mathrm{c}}^{2} R d t
$$

where $i_{\mathrm{c}}$ is the coil current, $R$ is the coil active resistance.

Power overloading can be expressed by:

$$
k_{\mathrm{po}}=\frac{A_{\mathrm{im}}(1-\eta)}{t_{\mathrm{cf}} \eta k_{\mathrm{h}}\left(\vartheta_{\mathrm{a}}-\vartheta_{0}\right) S_{\mathrm{c}}},
$$

where $A_{\mathrm{im}}$ is the head impact energy; $\eta$ is the electric drive emission; $\vartheta_{\mathrm{a}}$ is the allowable electric drive overheating temperature; $\vartheta_{0}$ is the ambient temperature.

With respect to (8) impact energy is:

$$
A_{\mathrm{im}}=\frac{t_{\mathrm{cf}} \eta k_{\mathrm{h}}\left(\vartheta_{\mathrm{a}}-\vartheta_{0}\right) S_{\mathrm{cs}}}{(1-\eta)} \cdot k_{\mathrm{po}}
$$

With respect to replacement in (9) by (6) and (7):

$$
\begin{gathered}
\left.A_{\mathrm{im}}\right|_{\min }=\frac{t_{\mathrm{cf}} \eta k_{\mathrm{h}}\left(\vartheta_{\mathrm{a}}-\vartheta_{0}\right) S_{\mathrm{cs}}}{(1-\eta)} \cdot \frac{\left(1-\varepsilon \gamma^{n}\right)(1-\gamma)}{(1-a)\left(1-\gamma^{n}\right)} \cdot \frac{a}{\gamma} \\
\left.A_{\mathrm{im}}\right|_{\max }=\frac{t_{\mathrm{cf}} \eta k_{\mathrm{h}}\left(\vartheta_{\mathrm{a}}-\vartheta_{0}\right) S_{\mathrm{cs}}}{(1-\eta)} \cdot \frac{\left(1-\varepsilon \gamma^{n}\right)(1-\gamma)}{(1-a)\left(1-\gamma^{n}\right)}
\end{gathered}
$$

If the heating mode is quasisteady and $n_{\max } \rightarrow \infty$, then (10) and (11) for allowable impact energy are reduced to:

$$
\begin{gathered}
A_{\mathrm{im} \max }=\frac{t_{\mathrm{cf}} \eta k_{\mathrm{h}}\left(\vartheta_{\mathrm{a}}-\vartheta_{0}\right) S_{\mathrm{cs}}}{(1-\eta)} \cdot \frac{(1-\gamma)}{(1-a)} \cdot \frac{a}{\gamma} ; \\
A_{\mathrm{im} \min }=\frac{t_{\mathrm{cf}} \eta k_{\mathrm{h}}\left(\vartheta_{\mathrm{a}}-\vartheta_{0}\right) S_{\mathrm{cs}}}{(1-\eta)} \cdot \frac{(1-\gamma)}{(1-a)} .
\end{gathered}
$$

According to (10) and (11), the equations with dimensionless parameters are derived: 


$$
\begin{aligned}
& A_{\mathrm{im} \min }^{*}=\frac{\left(1-\varepsilon \gamma^{n_{\max }}\right)(1-\gamma)}{(1-a)\left(1-\gamma^{n_{\max }}\right)} \cdot \frac{a}{\gamma}, \\
& A_{\mathrm{im} \max }^{*}=\frac{\left(1-\varepsilon \gamma^{n_{\max }}\right)(1-\gamma)}{(1-a)\left(1-\gamma^{n_{\max }}\right)} .
\end{aligned}
$$

Fig. 2 shows functions $A_{i m}^{*}=f\left(n_{\max }\right)$, found from (13).

$$
\text { If } T_{0} \gg t \text {, then } 1-\mathrm{e}^{-\frac{t}{T_{0}}} \approx \frac{t}{T_{0}} \text {. }
$$

The expressions (10) and (11) for impact energy are simplified:

$$
\begin{gathered}
A_{\mathrm{im}}\left(n_{\max }\right)_{\min }=\frac{\eta k_{\mathrm{h}}\left(\vartheta_{\mathrm{a}}-\vartheta_{0}\right) S_{\mathrm{sc}}}{(1-\eta)} \times \\
\times \frac{\left[1-\varepsilon\left(1-\frac{t_{\mathrm{c}}}{T_{0}} n_{\max }\right)\right]\left(T_{0}-t_{\mathrm{cp}}\right)}{n_{\max }} ; \\
A_{\mathrm{im}}\left(n_{\max }\right)_{\max }=\frac{\eta k_{\mathrm{T}}\left(\vartheta_{\mathrm{a}}-\vartheta_{0}\right) S_{\mathrm{cs}}}{(1-\eta)} \times \\
\times \frac{\left[1-\varepsilon\left(1-\frac{t_{\mathrm{c}}}{T_{0}} n_{\max }\right)\right] T_{0}}{n_{\max }} .
\end{gathered}
$$

If $\frac{t_{\mathrm{c}}}{T_{0}} n_{\max } \leq 0.1$, the computation error is less than $5 \%$.

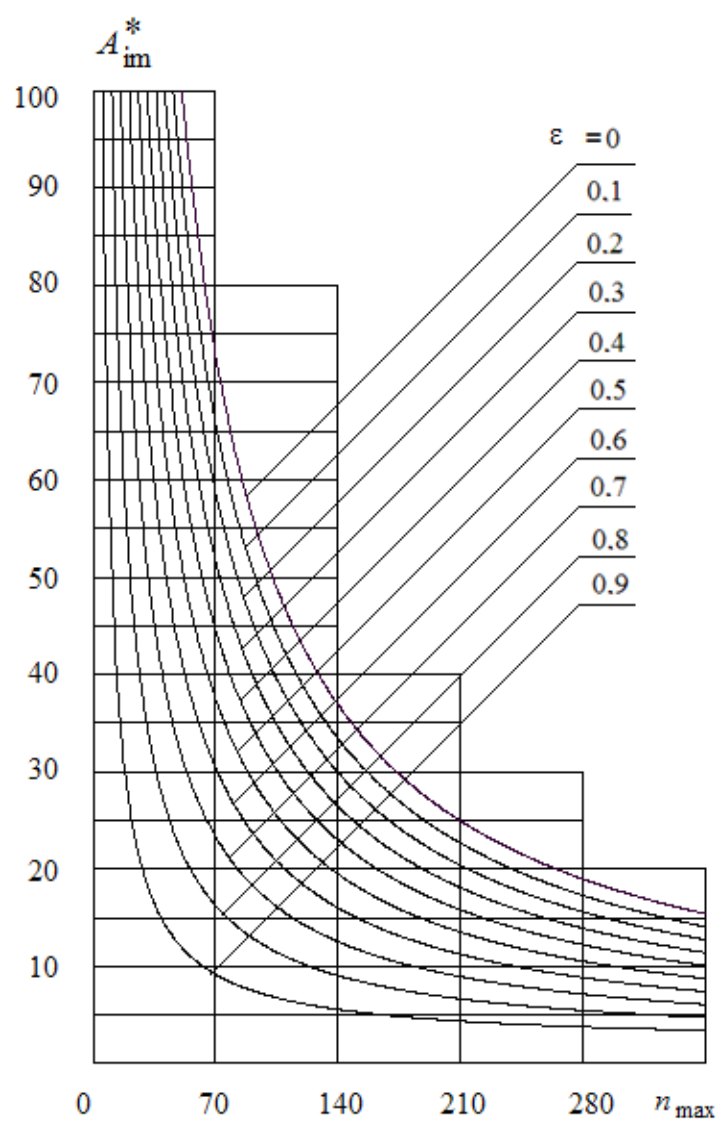

Fig. 2. Functions $A_{\mathrm{im}}^{*}(n)_{\max }=f(n)$ at $\frac{T_{0}}{t_{\mathrm{c}}}=2500$ and $\frac{t_{\mathrm{sf}}}{t_{\mathrm{c}}}=0.5$.

\section{III.ELECTRIC DRIVE DESIGN EXAMPLE}

For instance, it is necessary to build the regulation curve for the allowable impact energy $A_{\mathrm{im}}$ in the given range of executed working cycles (impacts) $n_{\max }=2300 \ldots 4800$.

When an electric drive is switched on, its overheating is $\vartheta_{\text {ini }}=95^{\circ} \mathrm{C}$.

The electric drive in Fig. 3 is powered by a half-wave rectifier from an $\mathrm{AC}$ source $50 \mathrm{~Hz}$, providing current pulses width $t_{\mathrm{cf}}=0,012 \mathrm{~s}$.

The electric drive parameters are $\eta=0.32, \vartheta_{0}=30^{\circ} \mathrm{C}$, $\vartheta_{\mathrm{a}}=125^{\circ} \mathrm{C}, \quad \vartheta_{\text {ini }}=95^{\circ} \mathrm{C}, \quad k_{\mathrm{h}}=12 \mathrm{~W} /\left(\mathrm{m}^{2} \cdot \mathrm{K}\right)$, $d_{1}=30 \mathrm{~mm}, d_{2}=75 \mathrm{~mm}, L_{\mathrm{c}}=42 \mathrm{~mm}, D=84 \mathrm{~mm}$, $L=100 \mathrm{~mm}, m_{\mathrm{EM}}=3.76 \mathrm{~kg}, T_{0}=2.94 \cdot 10^{3} \mathrm{~s}$.

The allowable impact energy in the given range of executed cycles is determined as: 
$A_{\text {im } \max }\left(n_{\max }\right)=\frac{2 \eta k_{\mathrm{h}} \tau_{\mathrm{a}} S_{\mathrm{cs}}}{1-\eta} \cdot \frac{\left[1-\varepsilon_{\tau}\left(1-\frac{t_{\mathrm{c}}}{T_{0}} n_{\max }\right)\right] T_{0}}{n_{\max }}=$

$$
=1.42 \cdot\left[1-0.684 \cdot\left(1-5.08 \cdot 10^{-6} n_{\max }\right)\right] \cdot \frac{2.94 \cdot 10^{3}}{n_{\max }} .
$$

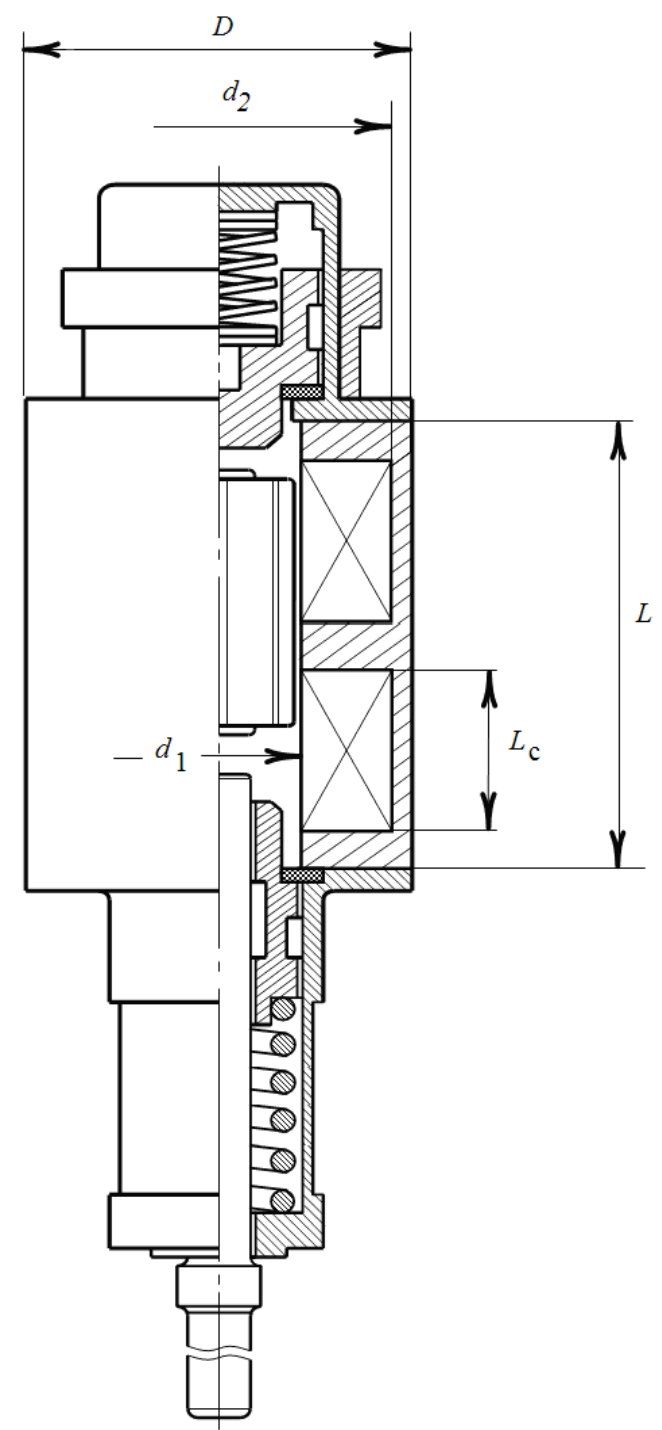

Fig. 3. Electromagnetic drive.

Cyclic electromagnetic drive and its design regulation curve are stated in Fig. 4.

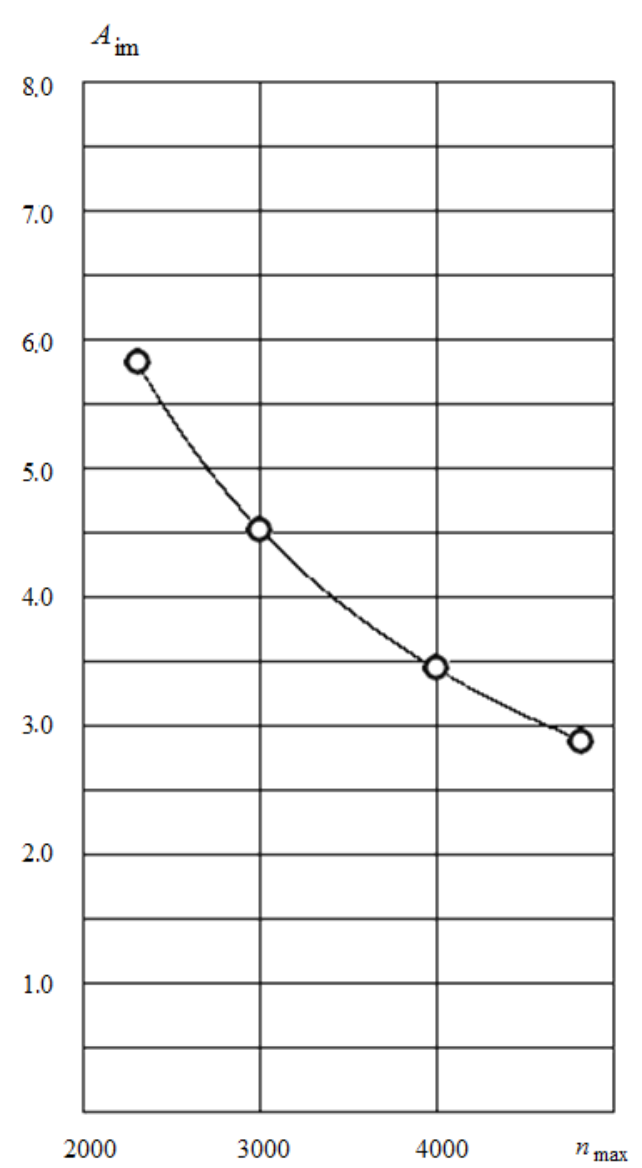

Fig. 4. Cyclic electromagnetic drive and its design regulation curve.

\section{IV.CONCLUSION}

The approximate design relations are derived to establish the allowable impact energy value depending on the executed working cycles number and initial temperature excess over the ambient temperature.

\section{References}

[1] K.M. Usanov, A.V. Volgin, E.A. Chetverikov, V.A. Kargin., A.P. Moiseev, Z.I. Ivanova, "Power electromagnetic strike machine for engineering-geological surveys", IOP Conference Series: Earth and Environmental Science [Electronic resource], p. 032049, 2017.

[2] B.F. Simonov, V.Y. Neyman, A.S. Shabanov, "Pulsed linear solenoid actuator for deep-well vibration source", Journal of Mining Science, vol. 53, Num. 1, pp. 117-125, 2017.

[3] K.M. Usanov, G.G. Ugarov, V.I. Moshkin, Linear impulse electromagnetic drive of machines with self-contained power supplies, Kurgan: Kurgan University, p. 284, 2006.

[4] A.A. Tatevosyan, A.S. Tatevosyan, "Calculation of magnetic system of the magnetoelectric machines", Dynamics of Systems, Mechanisms and Machines, Dynamics 2014 Proceedings, p. 7005698, 2014.

[5] O.B. Boule, Methods for calculating the magnetic systems of electric devices: magnetic circuit, field and program FEMM: Textbook, M.: Publishing. center «Academy», p. 336, 2005.

[6] Y.I. Klimenko, D.V. Batishchev, A.V. Pavlenko, V.P. Grinchenkov, "Design of a linear electromechanical actuator with an active vehicle 
[10] L.A. Neyman, V.Y. Neyman, K.A. Obukhov, "New method of the suspension system", Russian Electrical Engineering, vol. 86, Num. 10, pp. 588-593, 2015.

[7] R.R. Sattarov, "Electromechanical transients in passive suspension systems with eddy current dampers", 9th International Conference on Power Drives Systems, ICPDS 2016 - Conference Proceedings 9, p. 7756676, 2016.

[8] D.V. Batischev, A.V. Pavlenko, "On designing electromagnetic drives operating under conditions of high vibrations", Russian Electrical Engineering, vol. 83, Num. 8, pp. 423, 2012.

[9] L.A. Neyman, V.Y. Neyman, A.S. Shabanov, "Vibration dynamics of an electromagnetic drive with a half-period rectifier", The 18 international conference of young specialists on micro/nanotechnologies and electron devices, EDM 2017: proc., Altai, Erlagol, 29 June - 3 July 2017 , pp. 503-506, 2017 synchronous vibratory electromagnetic machine mechatronic module control", The 18 international conference of young specialists on micro/nanotechnologies and electron devices, EDM 2017: proc., Altai, Erlagol, 29 June - 3 July 2017. - Novosibirsk: NSTU, pp. 516-519, 2017.

[11] V.I. Moshkin, G.G. Ugarov, "The effect of pulse linear electromagnetic motors on voltage fluctuations in electric power networks of an industrial enterprise" Russian Electrical Engineering, vol. 86, Num. 2, pp. 61-65, 2015.

[12] K.M. Usanov, A.V. Volgin A.V, E.A. Chetverikov, V.A. Kargin, A.P., Moiseev, Z.I. Ivanova, "Power electromagnetic strike machine for engineering-geological surveys", IOP Conference Series: Earth and Environmental Science [Electronic resource], p. 032049, 2017. 